\title{
Activation of PAR-1/NADPH Oxidase/ ROS Signaling Pathways is Crucial for the Thrombin-Induced sFlt-1 Production in Extravillous Trophoblasts: Possible Involvement in the Pathogenesis of Preeclampsia
}

\author{
Qi-tao Huang $^{\mathrm{a}}$ Jian-hong Chen ${ }^{\mathrm{a}, \mathrm{b}} \quad$ Li-lin Hang ${ }^{\mathrm{a}}$ Shi-san Liu ${ }^{\mathrm{a}}$ Mei Zhong ${ }^{\mathrm{a}}$ \\ aDivision of Obstetrics and Gynecology, Nanfang Hospital, Southern Medical University, Guangzhou, \\ China; bepartment of Obstetrics and Gynecology, prenatal diagnostic center, Huizhou First Maternity \\ and Child Healthcare Hospital, Huizhou, China
}

\section{Key Words}

Thrombin • Trophoblast • sFlt-1

\begin{abstract}
Backgrounds/Aims: Preeclampsia was characterized by excessive thrombin generation in placentas and previous researches showed that thrombin could enhance soluble Fms-like tyrosine kinase 1 (sFlt-1) expression in first trimester trophoblasts. However, the detailed mechanism for the sFlt-1 over-production induced by thrombin was largely unknown. The purpose of this study was to explore the possible signaling pathway of thrombin-induced sFlt1 production in extravillous trophoblasts (EVT). Methods: An EVT cell line (HRT-8/SVneo) was treated with various concentrations of thrombin. The mRNA expression and protein secretion of sFlt- 1 in EVT were detected with real-time polymerase chain reaction and ELISA, respectively. The levels of intracellular reactive oxygen species (ROS) production were determined by DCFHDA. Results: Exposure of EVT to thrombin induced increased intracellular ROS generation and overexpression of sFlt-1 at both mRNA and protein levels in a dose dependent manner. Short interfering RNA (siRNA) directed against PAR-1 or apocynin (an inhibitor of NADPH oxidase) could decrease the intracellular ROS generation and subsequently suppressed the production of sFlt-1 at mRNA and protein levels. Conclusions: Our results suggested that thrombin increased sFlt-1 production in EVT via the PAR-1 /NADPH oxidase /ROS signaling pathway. This also highlights the PAR-1 / NADPH oxidase / ROS pathway might be a potential therapeutic target for the prevention of preeclampsia in the future.
\end{abstract}

Q.-t. Huang and J.-h. Chen contribute equally to this manuscript.

Mei Zhong, Professor

KARGER 125 


\section{Introduction}

Preeclampsia is a complex multi-system obstetric syndrome affecting about $5-10 \%$ of pregnant women and remains a leading cause of maternal and perinatal mortality and morbidity. It is characterized by hypertension and significant proteinuria at or after 20 weeks of pregnancy [1].

Dysregulations of angiogenic pathways have been showed to contribute to the inadequate blood supply of the placenta in patients with preeclampsia [2, 3]. Soluble Fms-like tyrosine kinase 1 (sFlt-1) is a splice variant of the vascular endothelial growth factor (VEGF) receptor Flt-1 that lacks the transmembrane and cytoplasmic domains [4]. Previous studies demonstrated that exogenous sFlt- 1 administered to pregnant rats could induce a preeclampsia-like phenotype including hypertension, proteinuria, and glomerular endotheliosis in these animals $[5,6]$. Moreover, circulating levels of sFlt-1 were elevated in the serum of women with preeclampsia [7-9], and this elevation preceded the development of clinical signs by 4-6 weeks. A recent pilot study performed by Thadhani et al. [10] found that lowering circulating sFlt- 1 by using extracorporeal apheresis was able to extend 3 preeclamptic pregnancies by 2-4 weeks, all of which resulted in healthy deliveries with no neonatal or maternal morbidity. Taken together, these observations suggested that excess sFlt-1 production might be associated with the development of preeclampsia.

Thrombin is a multifunctional serine protease generated at the site of vascular injury that transforms fibrinogen into fibrin, activates blood platelets and elicits multiple effects on a variety of cell types including extravillous trophoblast cells [11], endothelial cells [12], vascular smooth muscle cells [13], and monocytes [14]. It is widely known that uteroplacental hemorrhage [15], fibrin deposition [16], and infarction [17] are commonly observed in established preeclampsia. Moreover, uterine bleeding or the presence of hematoma at implantation sites in the first trimester is known to be associated with the later development of preeclampsia [18]. These conditions would generate excess thrombin. A recent research demonstrated that excess placental thrombin formation may be involved in the pathogenesis of preeclampsia [19]. A growing body of evidence demonstrated that plasma thrombin exerted its wide-range effect through activating specific proteinase-activated receptors (PAR, eg: PAR1, PAR3 and PAR4), NADPH oxidase system and inducing intracellular ROS production in various cell types [20-23]. For instance, prior studies suggested that endothelial dysfunction induced by excess formation of thrombin was associated with NADPH oxidase-dependent ROS generation. Inhibiting thrombin-PAR1 interaction or blocking NADPH oxidase activity could reduce the intracellular ROS generation and therefore improved endothelial function $[12,20]$.

Placenta was thought to be the primary source of circulating sFlt- 1 in women with preeclampsia. A previous study suggested that thrombin could enhance sFlt-1 expression in first trimester trophoblasts [11], establishing a possible link between thrombin and preeclampsia. However, the detailed mechanism for the sFlt-1 over-production caused by thrombin in placenta was largely unknown. The aim of the present study was to determine the possible role of the PAR-1/ NADPH oxidase signaling pathways of thrombin mediated sFlt-1 expression by trophoblasts.

\section{Materials and Methods}

The Institutional Review Board at the Nanfang Hospital, Southern Medical University approved the study protocol and all procedures.

Cell culture

First trimester EVT cell line (HTR-8/SVneo cells) was a kind gift from Dr Charles H. Graham (Queen's University, Ontario, Canada). The cells were cultured in RPMI-1640 (HyClone, South Logan, USA) supplemented with $10 \%$ fetal bovine serum (FBS), $100 \mathrm{U} / \mathrm{ml}$ penicillin, and $100 \mu \mathrm{g} / \mathrm{ml}$ streptomycin 


\section{Cellular Physiology Cell Physiol Biochem 2015;35:1654-1662

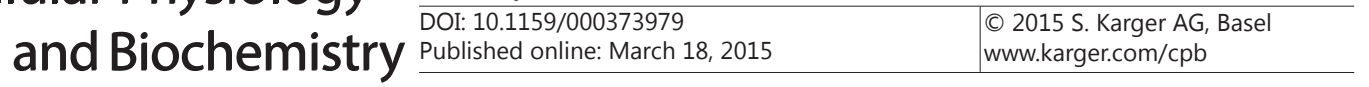 \\ Huang et al.: Mechanism of Thrombin Inducing sFlt-1 in Trophoblasts}

(HyClone), at $37{ }^{\circ} \mathrm{C}$ plus continuous supplement of $5 \% \mathrm{CO}_{2}$. Before experiments, HTR-8/SVneo cells were cultured in RPMI-1640 supplemented with 2.5\% FBS for $24 \mathrm{~h}$.

\section{Intervention with Cell Cultures}

EVT were rinsed with phosphate-buffered saline solution, replenished with fresh serum-free media, and cultured for an additional 24 hours. To evaluate the effect of thrombin on sFlt- 1 production in EVT, the cells were incubated with serum-free medium and different concentrations of thrombin $(0.1,1$, and $10 \mathrm{U} / \mathrm{ml}$; Sigma-Aldrich).We measured the intracellular ROS production of EVT treated with various concentrations of thrombin $(0.1,1$, and $10 \mathrm{U} / \mathrm{ml})$ in indicated time (12 hours) or thrombin $(10 \mathrm{U} / \mathrm{ml})$ over a time course $(3,6,12$ and 24 hours). In addition, we observed the effect of specific siRNA (inhibit different PAR receptors expression) or various potential enzymes[24] including inhibitors of NADPH oxidase (apocynin, 500 $\mu \mathrm{g} /$ $\mathrm{ml}$ ), nitric oxidase synthase(L-NNMA, $1000 \mu \mathrm{mol} / \mathrm{l}$ ), xanthine oxidase (allopurinol, $100 \mu \mathrm{mol} / \mathrm{l}$ ) and mitochondrial respiratory chain complexes(rotenone, $250 \mu \mathrm{mol} / \mathrm{l}$ ), on EVT in vitro and to explore whether specific siRNA or these enzymes may regulate the effect of thrombin on the expression of sFlt-1 in EVT.

\section{SiRNA transfection}

We used validated siRNA directed against PAR-1 (Cat. \# 4390771), PAR-3 (Cat. \# AM51331) and PAR4 (Cat. \# AM16708) mRNA from Ambion. Briefly, when EVT cells reached 70\%-85\% confluence, they were trypsinized and centrifuged at $90 \times \mathrm{g}$ at $4^{\circ} \mathrm{C}$ for $10 \mathrm{~min} .1 \times 10^{6}$ cells/condition were resuspended in $100 \mu \mathrm{L}$ of Nucleofector Solution (Amaxa/Lonza, Allendale, NJ) with $100 \mathrm{nmol} / \mathrm{L}$ siRNA. Electroporation transfection process was performed using the appropriate cell type program X005 in Nucleofector II (Amaxa Biosystems, Germany), as described previously [25]. After that, we immediately add $500 \mu$ of pre-warmed RPMI1640 with $10 \%$ FBS into the transfected cells, which were seeded into 6-well plates for 48 hours and then prepared for further analysis. Successful silencing of PAR-1, PAR-3, and PAR-4 were confirmed by Western blotting (Fig 1).

\section{RNA extraction, reverse transcription, and real-time quantitative polymerase chain Reaction}

Total RNA was extracted from EVT with the use of an RNeasy mini kit (Qiagen). One $\mu$ g total RNA was reverse transcribed in a $20 \mu \mathrm{L}$ volume with the use of ReverTraAcea (Toyobo) according to the manufacturer's instructions. Real-time quantitative polymerase chain reaction (PCR) and data analysis were performed using Lightcycler (Roche Diagnostic) according to the manufacturer's instructions. Two $\mu \mathrm{L}$ cDNA in $20 \mu \mathrm{L}$ volume was amplified with the use of oligonucleotide primers based on human sFlt-1 sequence. sFlt-1Primers (sense 5'-GCA CCT TGG TTG TGG CTG ACT-3', antisense 5'-GGG CCC GGG GGT CTC ATT ATT-3') were used to amplify a 643-bp product. The PCR conditions for the mRNA were as follows: 45 cycles at $95^{\circ} \mathrm{C}$ for 10 seconds, $63^{\circ} \mathrm{C}$ for 10 seconds, and $72^{\circ} \mathrm{C}$ for 27 seconds. All PCRs were followed with melting curve analysis. Relative gene expression was measured using $[\Delta \Delta c(t)]$ method and normalized to the geometric mean of two housekeeping genes: glycerinaldehyde dehydrogenase (GAPDH) (sense 5' - AGA TCA TCA GCA ATG CCT CC -3', antisense 5'- CAT GAG TCC TCC CAC GAT AC -3') and TATA-binding protein (TBP) (sense 5'- CAC GAA CCA CGG CAC TGA TT -3', antisense 5'- TTT TCT TGC TGC CAG TCT GGA C -3').

\section{Measurement of protein secretions in culture supernatants}

Conditioned culture media were centrifuged and stored at $-80^{\circ} \mathrm{C}$ until assay. Concentrations of sFlt- 1 in supernatants were measured with the use of its specific ELISA kits (Quantikine; R\&D Systems) according to the manufacturer's instructions.

\section{Measurement of intracellular ROS production}

The levels of intracellular ROS production were determined by measuring the fluorescence of 5 (and 6)-chloromethyl-2',7'-dichlorodrofluorescein diacetate (DCF, Molecular Probe, Carlsbad, CA). Briefly, EVT were pre-incubated for 30 min with $1 \mathrm{nmol} / \mathrm{L} \mathrm{DCF}$ in PBS without $\mathrm{Ca}^{2+}$ and $\mathrm{Mg}^{2+}$. The cells were then incubated with various concentrations of thrombin $(0.1,1$, and $10 \mathrm{U} / \mathrm{ml})$ [11] in indicated time (12 hours) or thrombin $(10 \mathrm{U} / \mathrm{ml})$ over a time course $(3,6,12$ and 24 hours) [24]. One milliter aliquots of the cells were removed for fluorescence intensity analysis on a flow cytometry (BD FACSCalibur system, Franklin Lakes, NJ). To verify the sources of ROS generation, EVT were pre-incubated for 10 mininuts with apocynin, L-NAME, allopurinol or rotenone as mentioned above (all from Sigma). 
Fig. 1. HTR-8/SVneo cells were transfected with PAR-1, PAR-3, PAR-4 siRNA or scramble siRNA (siNC). Western blot was performed to analyze the expression of endogenous PAR-1, PAR-3 and PAR-4 in HTR-8/SVneo cells. Glyceraldehyde-3-phosphate dehydrogenase (GAPDH) was used to verify equivalent loading. (* compared with control group, P < 0.05; \# compared with control group, $\mathrm{P}<0.01$ ).
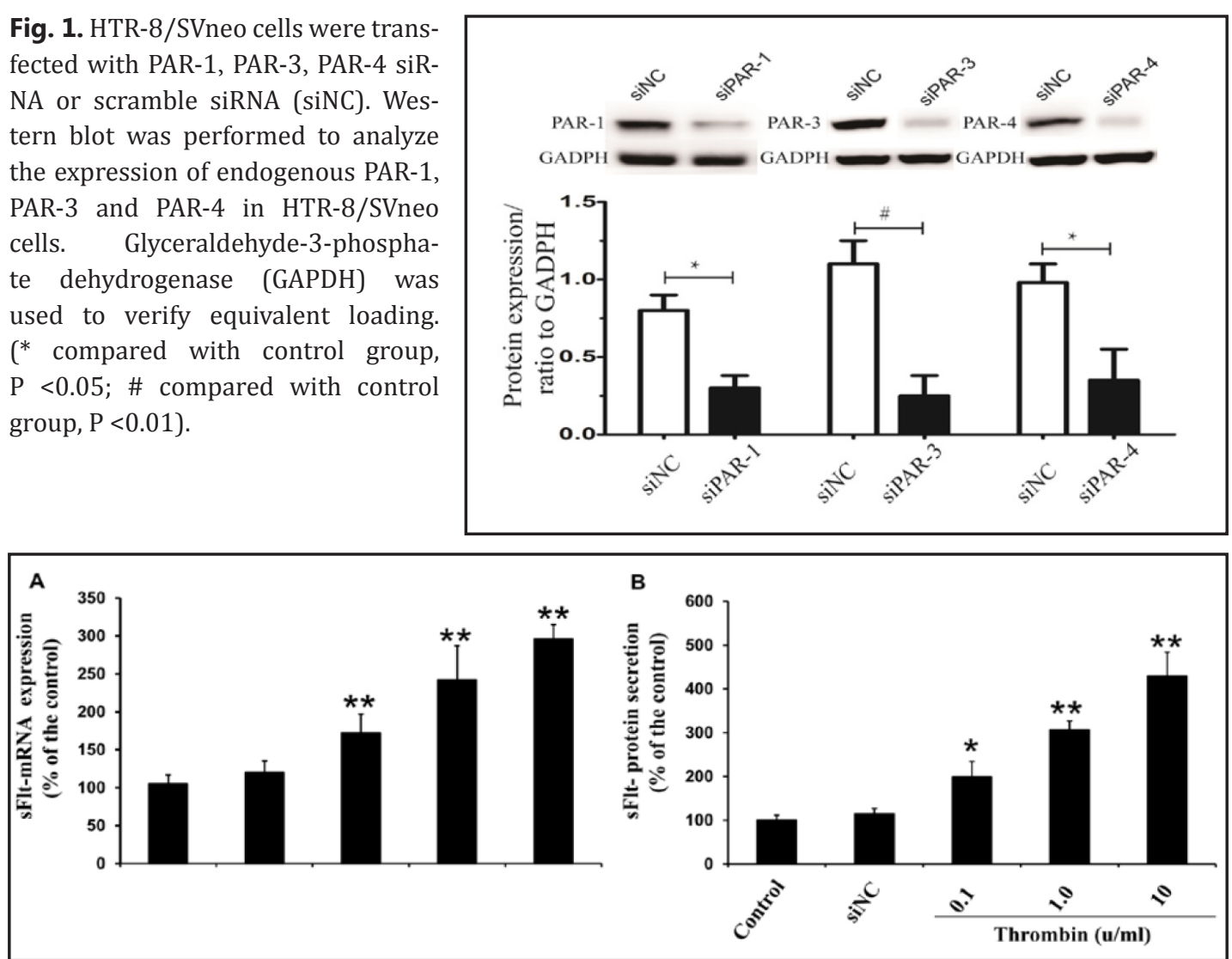

Fig. 2. (A)Effects of thrombin on sFlt-1 mRNA expression. (B) Effects of thrombin on on sFlt-1 secretion. Error bars depict SD. (* compared with control group, $\mathrm{P}<0.05$; ** compared with control group, $\mathrm{P}<0.01$ ).

Statistical Analysis

All experiments were performed 3 times or more independently and all the values were expressed as the mean \pm standard deviation (SD). Data were analyzed using one-way analysis of variance (ANOVA) with the application of the Dunnett's test. Differences were considered as statistically significant at $\mathrm{P}<0.05$.

\section{Results}

Effect of thrombin on sFlt-1 mRNA expression and protein secretion in EVT

As shown in Figure 2A\&B, exposure of EVT to thrombin significantly induced higher expressions of sFlt-1 at both the mRNA level and protein level in a dose dependent manner.

Thrombin increased intracellular ROS generation in EVT through PAR-1 / NADPH dependent pathway

Thrombin increased the intracellular ROS production in a time- and dose-dependent manner when compared with cells cultured in medium alone (Fig. 3A\&B). Furthermore, thrombin induced intracellular ROS production could be blocked by pretreating EVT with inhibitor of NADPH oxidase (apocynin), but not by the inhibitors of nitric oxidase synthase(LNNMA), xanthine oxidase(allopurinol) or the inhibitor of mitochondrial respiratory chain complexes(rotenone), suggesting that thrombin induced intracellular ROS production in EVT was dependent on NADPH oxidase activation (Fig. 3C). Moreover, specific siRNA was used to block the PARs expression in EVT. As shown in Fig. 3C, it was PAR-1 specific siRNA but not 


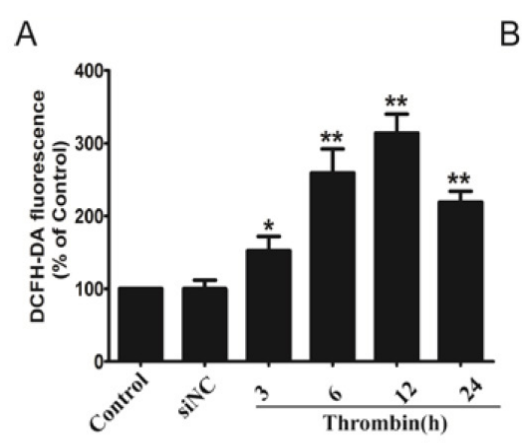

B

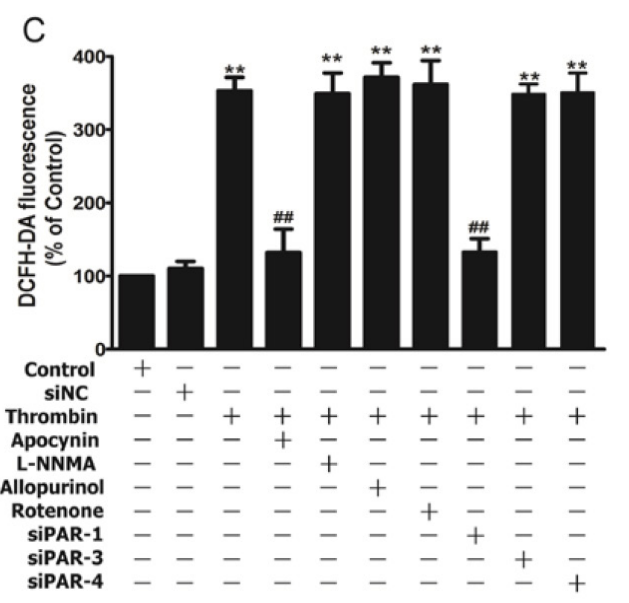

Fig. 3. Thrombin increased PAR-1 / NADPH-dependent intracellular ROS production in HTR-8/SVneo cells. Intracellular ROS production was assessed using DCFH-DA. (A) HTR-8/SVneo cells were treated with thrombin $(10 \mathrm{U} / \mathrm{ml})$ for $3,6,12$ and 24 hours. (B) HTR-8/SVneo cells were treated with various concentration of thrombin $(0.1,1.0,10 \mathrm{U} / \mathrm{ml})$ for 12 hours. (C) HTR-8/SVneo cells were transfected with PAR receptor or scramble siRNA (siNC) for $24 \mathrm{~h}$ or pretreated with apocynin (NADPH oxidase inhibitor, $500 \mu \mathrm{g} / \mathrm{ml}$ ), L-NNMA (nitric oxidase synthase $1000 \mu \mathrm{mol} / \mathrm{l}$ ), allopurinol (xanthine oxidase inhibitor, $100 \mu \mathrm{mol} / \mathrm{l}$ ), rotenone (inhibitor of mitochondrial electron transport complex I, $250 \mu \mathrm{mol} / \mathrm{l}$ ) for 10 minutes followed by incubation with thrombin $(10 \mathrm{U} / \mathrm{ml})$ for 12 hours. Data are representative of three independent experiments. $(*$ compared with control group, $\mathrm{P}<0.05$; ${ }^{* *}$ compared with control group, $\mathrm{P}<0.01$; \# compared with thrombin $(10 \mathrm{U} / \mathrm{ml})$ group, $\mathrm{P}<0.05$; \# \# compared with thrombin (10 U/ml) group, $\mathrm{P}<0.01)$.

other PARs siRNA or the scramble siRNA significantly blocked the enhanced ROS generation by thrombin, demonstrating that thrombin could bind PAR-1 to induce intracellular ROS generation in EVT.

Thrombin-induced sFlt-1 production in EVT was dependent on intracellular ROS generation via activation of PAR-1 and NADPH oxidase

We then examined whether intracellular ROS generation was necessary for thrombininduced sFlt-1 production in EVT. As demonstrated in Figure 4, both sFlt-1 mRNA expression and protein secretion in EVT treated with thrombin could be inhibited by apocynin in a does-dependent manner, but not by L-NNMA, allopurinol, rotenone. These data suggested that intracellular ROS generation through NADPH oxidase may be required for thrombininduced sFlt-1 production in EVT. In addition, thrombin induced sFlt-1 production in EVT could be also blocked by PAR-1 specific siRNA, but not other PARs siRNA or the scramble siRNA, indicating that thrombin induced sFlt-1 production was mainly mediated by a PAR-1 dependent intracellular ROS generation pathway. 
Fig. 4. (A) Effects of various inhibitors or siRNAs on thrombin(10 $\mathrm{U} / \mathrm{ml})$ induced sFlt-1 mRNA expression. (B) Effects of various inhibitors or siRNAs on thrombin(10 $\mathrm{U} / \mathrm{ml}$ )induced sFlt1 secretion. TH: thrombin $(10 \mathrm{U} / \mathrm{ml})$; apocynin (100; 250; 500): (NADPH oxidase inhibitor, $100 \mu \mathrm{g} / \mathrm{ml} ; 250$ $\mu \mathrm{g} / \mathrm{ml} ; 500 \mu \mathrm{g} / \mathrm{ml}$ ), L-NNMA: (nitric oxidase synthase $1000 \mu \mathrm{mol} / \mathrm{l}$ ), allopurinol: (xanthine oxidase inhibitor, $100 \mu \mathrm{mol} / \mathrm{l}$ ), rotenone: (inhibitor of mitochondrial electron transport complex I, $250 \mu \mathrm{mol} / \mathrm{l})$. Error bars depict SEM. (\# compared with control group, $\mathrm{P}<0.05$; \#\# compared with control group, $\mathrm{P}<0.01)$.

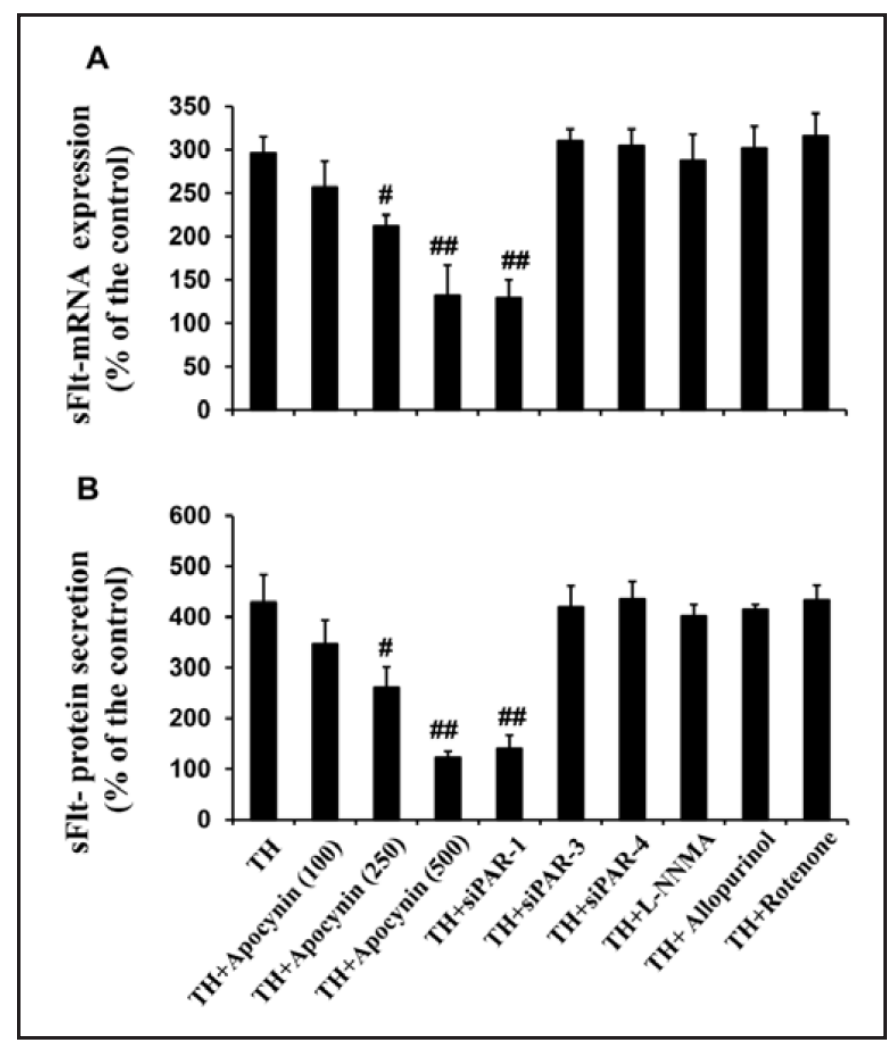

\section{Discussion}

Although abnormal sFlt-1 production is considered to be one of the crucial factors in the development of preeclampsia, the molecular signing pathways that regulate the production of sFlt-1 during preeclampsia are still not completely understood. In the present study, we demonstrated that thrombin increased the expression of sFlt-1 in trophoblasts by activation of PAR-1/NADPH oxidase/ROS signaling pathways and might thereby be involved in the development of preeclampsia.

Previous study showed that thrombin could activate intracellular signaling pathways by interacting with transmembrane domain G-protein-coupled receptors (Protease activated receptors, PARs). Four members of PARs have been cloned and designated PAR-1, PAR-2, PAR-3, and PAR-4 [26]. Three of these members, PAR-1, PAR-3, and PAR-4 were cleaved by thrombin, whereas PAR-2 was cleaved by trypsin. So one fundamental issue to be addressed was exactly how thrombin transmit their signals across the plasma membrane to elicit cellular activities. Erez et al. [27] showed that significantly stronger PAR-1 expression in the placenta of preeclampsia than their normal controls and suggested PAR-1 as a crucial mediator of thrombin induced coagulation and inflammation in preeclampsia. In this study, we provided evidence demonstrating that PAR-1 but not other PARs was required for thrombin-induced sFlt-1 production in EVT, since we could not inhibit thrombin-induced sFlt up-regulation by PAR3 and PAR4 receptor specific siRNA. These data suggested that PAR1 was involved in thrombin-induced sFlt expression and release from EVT.

In addition, a growing body of studies indicated that thrombin elicited its wide-range cellular effect through activating the PAR-1/NADPH oxidase system and inducing intracellular ROS in several different cell types [11-14, 20]. Mounting evidence suggested that PAR-1 participated actively in various vascular and inflammatory diseases [28, 29]. A recent study observed that protection effect against thrombin-induced endothelial dysfunction through inhibiting thrombin-PAR1 interaction [30].

Cariello et al. [23] showed that excess formation of thrombin was associated with NADPH oxidase-dependent ROS generation in hemodialysis patients and suggested that 
inhibition of NADPH oxidase may prevent thrombin-mediated damage in these patients. Here, we also observed that thrombin increased the intracellular ROS production in a timeand dose-dependent manner in EVT. Moreover, we demonstrated that apocynin (inhibitor of NADPH oxidase) but not other oxidase inhibitor, could decrease the intracellular ROS production and eventually inhibited thrombin-induced sFlt-1 expression, which suggested intracellular ROS generation by NADPH oxidase activation might be responsible for the thrombin-induced sFlt-1 expression.

Prior studies found that hypoxia and insufficient placentation, speculated to occur in the first trimester, could increase trophoblast sFlt-1 production, and caused consequently systemic and local endothelial dysfunction, which might induce further placental sFlt-1 production and enhanced placental hypoxia/ischemia, contributing to the 'vicious' cycle [5, 31]. Accumulating evidence also demonstrated that hypoxic conditions in the placenta could promote oxidative stress [5, 31,32]. Moreover, several recent researches have observed that oxidative stress could accelerate thrombin formation and enhance vascular reactivity to thrombin $[33,34]$. Thereby, excessive thrombin formation in the placenta might enhance sFlt-1 expression by trophoblasts and could be involved in this 'vicious' cycle.

One of the limitations in the present study was we only focused on the possible signaling pathways in EVT, a major cellular source of sFlt-1 in implantation sites. Prior studies have demonstrated that first trimester deciduae cells and peripheral blood monocytes could also express sFlt- $1[14,20]$. Thereafter, investigations were warranted to explore the possible role of thrombin on the sFlt-1expression in the decidua cells and peripheral blood monocytes in the near future.

In summary, the present study demonstrated that excess formation of thrombin might contribute to the pathogenesis of preeclampsia by promoting sFlt- 1 production in EVT, probably through activation of PAR-1/NADPH oxidase/ROS dependent pathway. We proposed understanding the possible mechanism of thrombin on EVT might be an important step toward development of new strategies and interventions for trophoblasts biological dysfunctions and preeclampsia. This also highlights the PAR-1 / NADPH oxidase / ROS pathway may be a potential therapeutic target for the prevention of preeclampsia in the future.

\section{Disclosure Statement}

The authors have no competing interests to declare.

\section{Acknowledgments}

The authors thank Dr Yun-fei Gao (University of Ottawa, Faculty of Medicine, Ontario, Canada) for reviewing the manuscript. This work was supported by the National Natural Science Foundation of China (81401208) to Dr Huang Qi-tao and the Science, Technology Planning Project of Guangdong Province (2013B021800142) to Dr Zhong Mei and the President Grant from Nanfang Hospital (2014B003). The funders had no role in study design, data collection and analysis, decision to publish, or preparation of the manuscript. We are appreciated the kind gift of first trimester extravillous trophoblast cell line (HTR-8/SVneo cells) provided from Dr. Charles H. Graham (Queen's University, Ontario, Canada). The study was approved by the local Institutional Review Board.

\section{References}

1 Steegers EA, von Dadelszen P, Duvekot JJ, Pijnenborg R: Preeclampsia. Lancet 2010;376:631-644.

2 Karumanchi SA, Bdolah Y: Hypoxia and sFlt-1 in preeclampsia: the "chicken-and-egg" question.

Endocrinology 2004;145:4835-4837. 


\section{Cellular Physiology Cell Physiol Biochem 2015;35:1654-1662 \begin{tabular}{ll|l} 
and Biochemistry & $\begin{array}{l}\text { DOI: 10.1159/000373979 } \\
\text { Published online: March 18, } 2015\end{array}$ & $\begin{array}{l}\text { C 2015 S. Karger AG, Basel } \\
\text { www.karger.com/cpb }\end{array}$ \\
\hline
\end{tabular} \\ Huang et al.: Mechanism of Thrombin Inducing sFlt-1 in Trophoblasts}

3 Oreshkova T, Dimitrov R, Mourdjeva M: A cross-talk of decidual stromal cells, trophoblast, and immune cells: a prerequisite for the success of pregnancy. Am J Reprod Immunol 2012;68:366-373.

4 Pfeffer PL, Pearton DJ: Trophoblast development. Reproduction 2012;143:231-246.

5 Maynard SE, Min JY, Merchan J, Lim KH, Li J, Mondal S, Libermann TA, Morgan JP, Sellke FW, Stillman IE, Epstein FH, Sukhatme VP, Karumanchi SA: Excess placental soluble fms-like tyrosine kinase 1 (sFlt1) may contribute to endothelial dysfunction, hypertension, and proteinuria in preeclampsia. J Clin Invest 2003;111:649-658.

6 Gilbert JS, Babcock SA, Granger JP: Hypertension produced by reduced uterine perfusion in pregnant rats is associated with increased soluble fms-like tyrosine kinase-1 expression. Hypertension 2007;50:11421147.

7 Smith GC, Crossley JA, Aitken DA, Jenkins N, Lyall F, Cameron AD, Connor JM, Dobbie R: Circulating angiogenic factors in early pregnancy and the risk of preeclampsia, intrauterine growth restriction, spontaneous preterm birth, and stillbirth. Obstet Gynecol 2007;109:1316-1324.

8 Kleinrouweler CE, Wiegerinck MM, Ris-Stalpers C, Bossuyt PM, van der Post JA, von Dadelszen P, Mol BW, Pajkrt E; EBM CONNECT Collaboration: Accuracy of circulating placental growth factor, vascular endothelial growth factor, soluble fms-like tyrosine kinase 1 and soluble endoglin in the prediction of preeclampsia: a systematic review and meta-analysis. BJOG 2012;119:778-787.

9 Masoura S, Kalogiannidis IA, Gitas G, Goutsioulis A, Koiou E, Athanasiadis A, Vavatsi N: Biomarkers in preeclampsia: a novel approach to early detection of the disease. J Obstet Gynaecol 2012;32:609-616.

10 Thadhani R, Kisner T, Hagmann H, Bossung V, Noack S, Schaarschmidt W, Jank A, Kribs A, Cornely OA, Kreyssig C, Hemphill L, Rigby AC, Khedkar S, Lindner TH, Mallmann P, Stepan H, Karumanchi SA, Benzing T: Pilot study of extracorporeal removal of soluble fms-like tyrosine kinase 1 in preeclampsia. Circulation 2011;124:940-950.

11 Zhao Y, Koga K, Osuga Y, Nagai M, Izumi G, Takamura M, Harada M, Hirota Y, Yoshino O, Taketani Y: Thrombin enhances soluble Fms-like tyrosine kinase 1 expression in trophoblasts; possible involvement in the pathogenesis of preeclampsia. Fertil Steril 2012;98:917-921.

12 Leonard A, Marando C, Rahman A, Fazal F: Thrombin selectively engages LIM kinase 1 and slingshot-1L phosphatase to regulate NF- $\mathrm{BB}$ activation and endothelial cell inflammation. Am J Physiol Lung Cell Mol Physiol 2013;305:651-664.

13 Popović M, Smiljanić K, Dobutović B, Syrovets T, Simmet T, Isenović ER: Thrombin and vascular inflammation. Mol Cell Biochem 2012;359:301-313.

14 Rajakumar A, Michael HM, Rajakumar PA, Shibata E, Hubel CA, Karumanchi SA, Thadhani R, Wolf M, Harger G, Markovic N: Extra-placental expression of vascular endothelial growth factor receptor-1, (Flt-1) and soluble Flt-1 (sFlt-1), by peripheral blood mononuclear cells (PBMCs) in normotensive and preeclamptic pregnant women. Placenta 2005;26:563-573.

15 Lockwood CJ, Huang SJ, Krikun G, Caze R, Rahman M, Buchwalder LF, Schatz F: Decidual hemostasis, inflammation, and angiogenesis in preeclampsia. Semin Thromb Hemost 2011;37:158-164.

16 Pinheiro MB, Gomes KB, Dusse LM: Fibrinolytic system in preeclampsia. Clin Chim Acta 2013;416:67-71.

17 Stevens DU, Al-Nasiry S, Bulten J, Spaanderman ME: Decidual vasculopathy in preeclampsia: lesion characteristics relate to disease severity and perinatal outcome. Placenta 2013;34:805-809.

18 Smits LJ, North RA, Kenny LC, Myers J, Dekker GA, McCowan LM: Patterns of vaginal bleeding during the first 20 weeks of pregnancy and risk of preeclampsia in nulliparous women: results from the SCOPE study. Acta Obstet Gynecol Scand 2012;91:1331-1338.

19 Xin H, Zhang Y, Wang H, Sun S: Alterations of profibrinolytic receptor annexin A2 in preeclampsia: a possible role in placental thrombin formation. Thromb Res 2012;129:563-567.

20 Lockwood CJ, Toti P, Arcuri F, Norwitz E, Funai EF, Huang ST, Buchwalder LF, Krikun G, Schatz F: Thrombin regulates soluble fms-like tyrosine kinase-1 (sFlt-1) expression in first trimester decidua: implications for preeclampsia. Am J Pathol 2007;170:1398-405.

21 García-López MT, Gutiérrez-Rodríguez M, Herranz R: Thrombin-activated receptors: promising targets for cancer therapy? Curr Med Chem 2010;17:109-128.

22 Sokolova E, Reiser G: Prothrombin/thrombin and the thrombin receptors PAR-1 and PAR-4 in the brain: localization, expression and participation in neurodegenerative diseases. Thromb Haemost 2008;100:576581. 


\section{Cellular Physiology Cell Physiol Biochem 2015;35:1654-1662

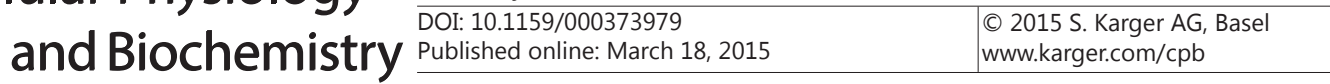 \\ Huang et al.: Mechanism of Thrombin Inducing sFlt-1 in Trophoblasts}

23 Cariello M, Simone S, Loverre A, Gigante M, Incampo F, Pietanza S, Colucci M, Schena FP, Gesualdo L, Grandaliano G, Pertosa G: Coagulation activation is associated with nicotinamide adenine dinucleotide phosphate oxidase-dependent reactive oxygen species generation in hemodialysis patients. Antioxid Redox Signal 2012;16:428-439.

24 Huang QT, Zhang M, Zhong M, Yu YH, Liang WZ, Hang LL, Gao YF, Huang LP, Wang ZJ: Advanced glycation end products as an upstream molecule triggers ROS-induced sFlt-1 production in extravillous trophoblasts: a novel bridge between oxidative stress and preeclampsia. Placenta 2013;34:1177-1182.

25 Forbes K, Desforges M, Garside R, Aplin JD, Westwood M: Methods for siRNA-mediated reduction of mRNA and protein expression in human placental explants, isolated primary cells and cell lines. Placenta 2009;30:124-129.

26 Vidwan P, Pathak A, Sheth S, Huang J, Monroe DM, Stouffer GA: Activation of protease-activated receptors 3 and 4 accelerates tissue factor-induced thrombin generation on the surface of vascular smooth muscle cells. Arterioscler Thromb Vasc Biol 2010;30:2587-2596.

27 Erez O, Romero R, Kim SS, Kim JS, Kim YM, Wildman DE, Than NG, Mazaki-Tovi S, Gotsch F, Pineles B, Kusanovic JP, Espinoza J, Mittal P, Mazor M, Hassan SS, Kim CJ: Over-expression of the thrombin receptor (PAR-1) in the placenta in preeclampsia: a mechanism for the intersection of coagulation and inflammation. J Matern Fetal Neonatal Med 2008;21:345-355.

28 Zhu W, Chandrasekharan UM, Bandyopadhyay S, Morris SM Jr, DiCorleto PE, Kashyap VS: Thrombin induces endothelial arginase through AP-1 activation. Am J Physiol Cell Physiol 2010;298:952-960.

29 Chapman J: Coagulation in inflammatory diseases of the central nervous system. Semin Thromb Hemost 2013;39:876-880.

30 Kaneider NC, Leger AJ, Agarwal A, Nguyen N, Perides G, Derian C, Covic L, Kuliopulos A: 'Role reversal' for the receptor PAR1 in sepsis-induced vascular damage. Nat Immunol 2007;8:1303-1312.

31 Murphy SR, LaMarca BB, Parrish M, Cockrell K, Granger JP: Control of soluble fms-like tyrosine-1 (sFlt-1) production response to placental ischemia/hypoxia: role of tumor necrosis factor- $\alpha$. Am J Physiol Regul Integr Comp Physiol 2013;304:130-135.

32 Laresgoiti-Servitje E, Gomez-Lopez N: The pathophysiology of preeclampsia involves altered levels of angiogenic factors promoted by hypoxia and autoantibody-mediated mechanisms. Biol Reprod 2012;87:36.

33 Gajos G, Zalewski J, Rostoff P, Nessler J, Piwowarska W, Undas A: Reduced thrombin formation and altered fibrin clot properties induced by polyunsaturated omega-3 fatty acids on top of dual antiplatelet therapy in patients undergoing percutaneous coronary intervention (OMEGA-PCI clot). Arterioscler Thromb Vasc Biol 2011;31:1696-1702.

34 Kameda K, Kikkawa Y, Hirano M, Matsuo S, Sasaki T, Hirano K: Combined argatroban and anti-oxidative agents prevents increased vascular contractility to thrombin and other ligands after subarachnoid haemorrhage. Br J Pharmacol 2012;165:106-119. 